\title{
Optimal Impact Angle Guidance for Exo-Atmospheric Interception Utilizing Gravitational Effect
}

\author{
Shaoming He and Chang-Hun Lee*
}

\begin{abstract}
This paper aims to develop a new optimal intercept angle guidance law for exo-atmospheric interception by utilizing gravity. A finite-time optimal regulation problem is formulated by considering the instantaneous zero-effort-miss (ZEM) and the intercept angle error as the system states. The analytical guidance command is then derived based on Schwarz's inequality approach and Lagrange multiplier concept. Capturability analysis using instantaneous linear time-invariant system concept is also presented to provide better insights of the proposed guidance law. Theoretical analysis reveals that the proposed optimal guidance law encompasses previously suggested optimal impact angle constrained guidance laws. Numerical simulations with some comparisons clearly demonstrate the effectiveness of the proposed guidance law.
\end{abstract}

Index Terms-Missile guidance, Intercept angle, Optimal control, Varying-speed, Gravity

\section{INTRODUCTION}

The ability to constrain the final intercept angle of the antiship or anti-tank missile is often desirable for increasing the warhead effectiveness as well as the kill probability since it enables the missile to attack a vulnerable or weak point on a target [1]-[8]. For other types of homing missile systems, the interception angle control is required to maintain advantageous homing engagement geometry against a target as well as to ensure a better quality of seeker measurement on a target [9][11]. For these reasons, over the past decades, extensive efforts have been made in the area of intercept angle or impact angle control for tactical missiles.

Until recently, the optimal control theory has been widely used to devise advanced intercept angle guidance laws due to its well-established design process and ability to satisfy terminal constraints by a state feedback formulation. Optimal guidance laws bring in the philosophy of trajectory shaping by optimizing a meaningful cost function as well as meeting certain terminal boundary conditions [12]. In [13], the authors first proposed an optimal impact angle control guidance law in the vertical plane for reentry vehicles. In [14], the authors suggested a numerical solution to achieve vertical interception with minimum interception time to increase the attacking missile's survivability. Zarchan [15] derived a trajectory shaping guidance (TSG) law to cater for the intercept angle by minimizing the energy consumption. Later in [16], the TSG

Shaoming He and Chang-Hun Lee are with the School of Aerospace, Transport and Manufacturing, Cranfield University, Cranfield MK43 OAL, UK.

*Corresponding Author. Email: lckdgns@gmail.com was extended to missiles with arbitrary order control system, i.e., autopilot, and the closed-form solution was also provided. Linear quadratic optimal guidance law and optimal differential game guidance laws that explicitly enable imposing a predetermined intercept angle were proposed in [17]. To provide one more degree-of-freedom of trajectory shaping, a $n$th order time-to-go weighted cost function was utilized in [18], [19] to design optimal guidance laws for impact angle control. Lee et al. [20] further developed a generalized optimal guidance law with arbitrary weighting functions to satisfy terminal angle constraint.

Note that most previous optimal intercept angle guidance laws were designed by assuming that the vehicle moves with constant velocity. Generally, for most homing missile systems, the interception event takes place during gliding phase (absence of axial acceleration due to thrust) and the homing duration is relatively short compared to other flight phases. Therefore, the speed of the interceptor can be considered as a slow-varying variable and the constant velocity assumption is valid. However, for an exo-atmospheric scenario, where aerodynamic force is not available to control an interceptor, thrust vector control is widely used as an alternative way and the interceptor velocity increases due to the presence of axial acceleration [10], [21]. In this case, obviously, the constant velocity assumption is not valid and classical optimal impact angle guidance laws, which relies on the constant-speed assumption, cannot drive the missile to follow the desired interception course and is far away from optimality. Optimal guidance laws that addressed the issue of varying missile velocity can be found in [10], [22]-[25], but terminal flight path angle was not constrained in these guidance laws. In a recent noteworthy contribution [26], the authors provided a solution to design impact angle guidance law for speed-varying missiles. However, this guidance law was limited to address the optimality issue because it was formulated based on a kind of nonlinear control approach rather than the optimal control approach.

Another issue of previous optimal intercept angle guidance laws is that most of them were proposed under the gravityfree assumption except for some computational guidance algorithms [27], [28]. The gravity-free assumption justifies the linearization around the straight line collision course and therefore simplifies optimal guidance law derivation process. When implementing these guidance laws in reality, an additional term is utilized to counteract the effect of gravitational acceleration. This simple and straightforward method, obviously, requires 
more control energy and cannot guarantee zero final guidance command, leading to the sacrifice of the direct hit effectiveness as well as the operational margins. Especially, these issues become more significant in the case of exo-atmospheric interception. Notice that practical interceptors usually have constrained maneuverability [29], [30]. Leveraging simple gravity compensation is not a wise option.

Motivated by the above observations, this paper aims to propose a new optimal intercept angle guidance law for accelerating missiles in exo-atmospheric interception by utilizing gravity. In our previous work [25], we suggested a new gravityturn-assisted optimal guidance law that directly uses, instead of compensating for, the gravity for accelerating missiles. This paper extends [25] to optimal intercept angle guidance. To achieve this, we first formulate a finite-time regulation problem by considering instantaneous zero-effort-miss ZEM [25] and intercept angle as the system states to be controlled. The proposed guidance law is then analytically derived through Schwarz's inequality approach with the aid of Lagrange multiplier concept. The final guidance command is given in a feedback form with time-varying navigation gains. Theoretical analysis shows that the navigation gains gradually converge to constant values when the missile approaches the target. Without the consideration of gravity and axial acceleration, the proposed guidance law reduces to classical time-to-go weighted optimal impact angle guidance (TWOIAG), proposed in [18], [19].

The key features of the proposed guidance law are twofold. On one hand, the gravity-turn concept is leveraged in guidance law derivation and thus the proposed algorithm requires no additional term to counteract the gravitational effect. This strategy enables energy saving and accurate interception, as confirmed by numerical simulation results. On the other hand, the commended acceleration of the proposed guidance law converges to zero at the time of impact. Therefore, the proposed approach provides operational margins and improves robustness against external uncertainties, especially near the interception.

The rest of the paper is organized as follows. Sec. II presents some preliminaries and backgrounds of this paper. Sec. III derives the proposed optimal intercept angle guidance law, followed by the capturability analysis and discussion of the proposed guidance law shown in Sec. IV. Finally, some numerical simulations and conclusions are offered.

\section{PRELIMINARIES AND BACKGROUNDS}

This section states some preliminary backgrounds on the kinematics model and the instantaneous ZEM concept in the exo-atmospheric guidance problem. The problem formulation of this paper is also presented.

\section{A. Missile-Target Kinematics}

This paper considers the planar exo-atmospheric homing engagement geometry, shown in Fig. 1, where $M$ and $T$ denote the missile and the target, respectively. The coordinate $X_{I} O Y_{I}$ represents the inertial frame. $r$ is the relative distance between the interceptor and the target. The notations $\lambda$ and $\gamma$ denote the

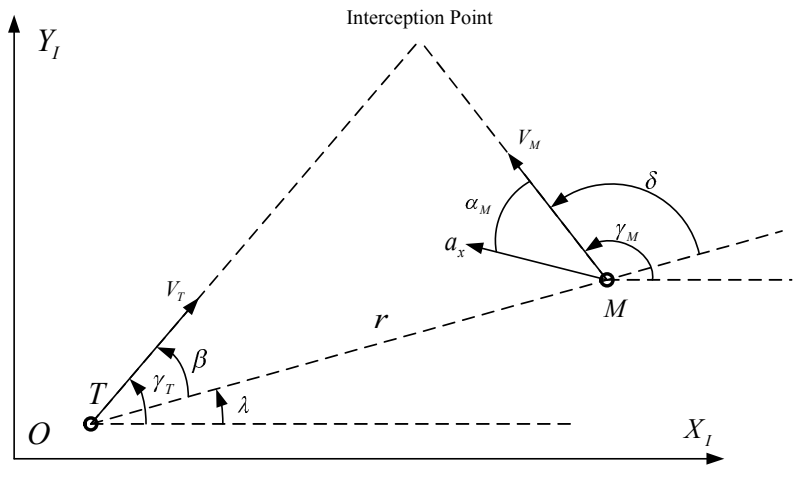

Fig. 1. Planar geometry of homing engagement.

line-of-sight (LOS) angle and flight path angle. $V$ is velocity of the vehicle. The variable of $a_{x}$ stands for the missile's axial acceleration. $\alpha_{M}$ is the interceptor's shear angle, which is the angle between the velocity vector and thrust vector for exoatmospheric vehicles [31]. In order to allow for a closed-form formulation, we assume that the missile's axial acceleration $a_{x}$, provided by a mounted rocket motor, is constant. Note that this assumption is widely-accepted in guidance law design for accelerating missiles [10], [21].

The differential equations describing the missile-target relative kinematics are formulated as

$$
\begin{gathered}
\dot{r}=V_{r} \\
r \dot{\lambda}=V_{\lambda}
\end{gathered}
$$

where the relative speeds along and perpendicular to the LOS are

$$
\begin{aligned}
& V_{r}=V_{M} \cos \delta-V_{T} \cos \beta \\
& V_{\lambda}=V_{M} \sin \delta-V_{T} \sin \beta
\end{aligned}
$$

The complementary equations that determine the relationship between the lead angle and flight path angle are

$$
\begin{gathered}
\delta=\gamma_{M}-\lambda \\
\beta=\gamma_{T}-\lambda
\end{gathered}
$$

During the exo-atmospheric endgame, there is no aerodynamic force and the flight path angle is only controlled by the normal acceleration $a_{M}$ which is introduced by the axial acceleration and the shear angle as

$$
a_{M}=a_{x} \sin \alpha_{M}
$$

The interceptor employs an attitude control system to change the direction of the thrust, that is $\alpha_{M}$, to generate the required normal acceleration for trajectory shaping and to provide energy increasing [10], [21]. For simplicity, this paper assumes that the attitude control system is ideal, i.e., the desired attitude is obtained with no time delays. Note that this assumption is valid with the purpose of deriving a guidance law because the control loop is usually designed to be fast enough with respect to the guidance loop. Then, due to the 
missile's acceleration and gravitational effect, the flight path angle and speed of the vehicle evolve according to

$$
\begin{gathered}
\dot{\gamma}_{M}=\frac{a_{M}-g \cos \gamma_{M}}{V_{M}} \\
\dot{V}_{M}=a_{x} \cos \alpha_{M}-g \sin \gamma_{M}
\end{gathered}
$$

where $g$ stands for the gravitational acceleration, which is assumed as a constant because the duration of the terminal guidance phase is typically very short.

\section{B. Instantaneous Zero-Effort-Miss}

In order to intercept a target, the primary control interest of exo-atmospheric interception is to design a guidance law to nullify the heading error or the equivalent ZEM. For exoatmospheric interception, it is also desirable to reduce the magnitude of interceptor's shear angle $\alpha_{M}$ as much as possible so as to increase the impact energy as well as to maximize the effectiveness of direct hit. This requirement is equivalent to reduce the final guidance command because for a given axial acceleration the normal acceleration is only proportional to the shear angle as shown in Eq. (7).

Note that most previous exo-atmospheric guidance laws assumed no gravity in the design process and leveraged an additional add-on term $g \cos \gamma_{M}$ to reject the effect of gravity in implementation. This straightforward gravity compensation approach works well in implementation, but requires more energy and obviously cannot guarantee zero final guidance command, leading to the sacrifice of the effectiveness of direct hit and the operational margins. In order to tackle this problem, a gravity-turn-assisted optimal guidance law that directly uses, instead of compensating for, the gravity for exo-atmospheric interception guidance was proposed in our previous work [25]. Unlike the gravity-free case, the desired collision course from the missile to the predicted interception point (PIP), when utilizing gravity, is a curved line, and therefore directly minimizing the ZEM is intractable. To accommodate this issue, we introduced a new concept, called instantaneous ZEM, in [25] to enable analytical derivation of the optimal guidance law. The instantaneous ZEM is defined as the final distance the missile would miss the target if the target continues along its present course and the missile follows a straight line along its current flight path angle with no further corrective maneuvers. The geometric interpretation of the proposed instantaneous ZEM is shown in Fig. 2, where the curved path $M-P I P$ is the desired trajectory, $M-A$ the uncontrolled flight path from the current time onward and the straight line $M-B$ the instantaneous uncontrolled flight trajectory. Note that the original ZEM converges to zero as the instantaneous ZEM approaches zero. Therefore, zeroing instantaneous ZEM allows for the interception of a target.

According to [25], the instantaneous ZEM $z$ and its rate $\dot{z}$ can be obtained as

$$
\begin{gathered}
z=-e_{\gamma}\left(V_{M} t_{g o}+\frac{1}{2} a_{x} t_{g o}^{2}\right) \\
\dot{z}=-a_{M}\left(t_{g o}+\frac{a_{x}}{2 V_{M}} t_{g o}^{2}\right)
\end{gathered}
$$

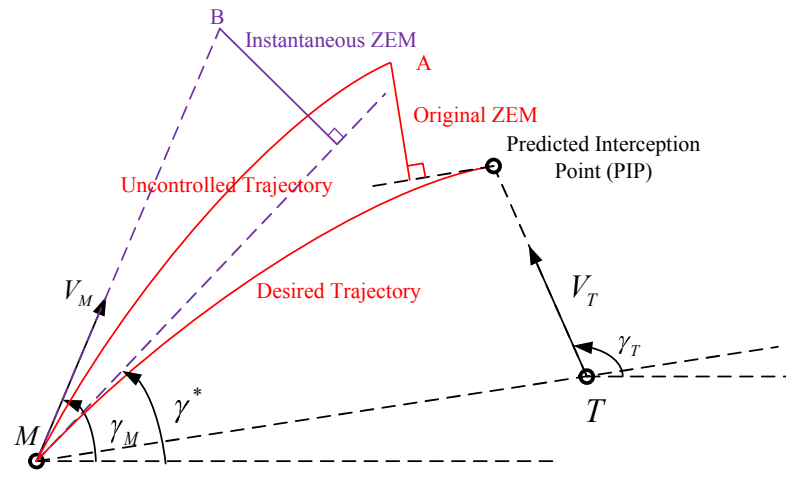

Fig. 2. Definition of instantaneous ZEM.

where $e_{\gamma}=\gamma_{M}-\gamma^{*}$ is the heading error with $\gamma^{*}$ being the desired current flight path angle calculated from the collision triangle, and $t_{g o}$ the remaining flight time calculated from the geometry. Detailed explanation of how to derive the desired collision triangle and time-to-go for gravity-assisted-turn case can be found in [25]. The prediction of future interceptor's trajectory for the exo-atmospheric case, which is required to calculate the desired collision triangle, can be found in [32].

Note that the collision triangle, derived in [25], automatically contains the gravity and therefore one only needs to use $\dot{\gamma}=a_{M} / V_{M}$ in guidance law design.

\section{Problem Formulation}

The analysis in [25] revealed that nullifying the instantaneous ZEM renders perfect interception that directly uses the gravitational effect. Additionally, constraining the intercept angle is desirable in terms of increasing the direct hit effectiveness as well as the kill probability for exo-atmospheric interception in practice. Therefore, the aim of this paper is to design a new optimal guidance law that drives both instantaneous ZEM and intercept angle error to converge to zero simultaneously at the time of impact. The intercept angle here is defined as the missile's flight path angle at the time of impact.

\section{Derivation of the Optimal Intercept Angle GUIDANCE LAW}

In this section, the proposed optimal guidance law is derived by using optimal control theory. In order to intercept the target with desired impact angle $\gamma_{f}^{d}$, we formulate the following finite-time optimal control problem

$$
\min _{a_{M}} J=\frac{1}{2} \int_{t}^{t_{f}} R(\tau) a_{M}^{2}(\tau) d \tau
$$

subject to

$$
\begin{aligned}
& \dot{z}=-a_{M}\left(t_{g o}+\frac{a_{x}}{2 V_{M}} t_{\text {go }}^{2}\right), \quad z\left(t_{f}\right)=0 \\
& \dot{\gamma}_{M}=\frac{a_{M}}{V_{M}}, \quad \gamma_{M}\left(t_{f}\right)=\gamma_{f}^{d}
\end{aligned}
$$

where $R(t)>0$ is an arbitrary weighting function.

As shown in Eq. (13), the system equation contains the timevarying nonlinear term due to the time-to-go (i.e., $t_{g o}$ ) and 
missile velocity. Accordingly, because of such nonlinearity, it is relatively difficult to apply the standard optimal control theory to find the analytical guidance command. We thereby seek to solve the preceding optimal control problem through Schwarz's inequality approach. According to the linear control theory, the general solution of the guidance system (13) can be expressed as

$$
\begin{aligned}
& z\left(t_{f}\right)=z-\int_{t}^{t_{f}} h_{1}(\tau) a_{M}(\tau) d \tau \\
& \gamma_{M}\left(t_{f}\right)=\gamma_{M}-\int_{t}^{t_{f}} h_{2}(\tau) a_{M}(\tau) d \tau
\end{aligned}
$$

where

$$
h_{1} \triangleq\left(t_{g o}+\frac{a_{x}}{2 V_{M}} t_{g o}^{2}\right), \quad h_{2} \triangleq-\frac{1}{V_{M}}
$$

Imposing the terminal constraints on Eq. (14) gives

$$
\begin{array}{r}
f_{1} \triangleq z=\int_{t}^{t_{f}} h_{1}(\tau) a_{M}(\tau) d \tau \\
f_{2} \triangleq \gamma_{M}-\gamma_{f}^{d}=\int_{t}^{t_{f}} h_{2}(\tau) a_{M}(\tau) d \tau
\end{array}
$$

Obviously, in the considered problem, two constraints are required to be satisfied with only one control input. To this end, a Lagrange multiplier $\lambda$ is introduced to merge these two constraints as

$$
f_{1}-\lambda f_{2}=\int_{t}^{t_{f}}\left[h_{1}(\tau)-\lambda h_{2}(\tau)\right] a_{M}(\tau) d \tau
$$

Introducing a slack variable $R(t)$ renders Eq. (17) to

$$
\begin{aligned}
& f_{1}-\lambda f_{2} \\
& =\int_{t}^{t_{f}}\left[h_{1}(\tau)-\lambda h_{2}(\tau)\right] R^{-1 / 2}(\tau) R^{-1 / 2}(\tau) a_{M}(\tau) d \tau
\end{aligned}
$$

Applying the Schwarz's inequality to the preceding equation yields

$$
\begin{array}{r}
\frac{\left(f_{1}-\lambda f_{2}\right)^{2}}{2 \int_{t}^{t_{f}}\left[h_{1}(\tau)-\lambda h_{2}(\tau)\right]^{2} R^{-1}(\tau) d \tau} \\
\leq \frac{1}{2} \int_{t}^{t_{f}} R^{-1}(\tau) a_{M}^{2}(\tau) d \tau
\end{array}
$$

which gives a lower bound of the performance index. According to the Schwarz's inequality, the equality of Eq. (19) holds if and only if there exists a constant $C$ such that

$$
a_{M}(\tau)=C\left[h_{1}(\tau)-\lambda h_{2}(\tau)\right] R^{-1}(\tau)
$$

Substitution of Eq. (20) into Eq. (16) results in

$$
f_{1}=C \int_{t}^{t_{f}} h_{1}(\tau)\left[h_{1}(\tau)-\lambda h_{2}(\tau)\right] R^{-1}(\tau) d \tau
$$

Solving Eq. (21) for $C$ gives

$$
C=\frac{f_{1}}{\int_{t}^{t_{f}} h_{1}(\tau)\left[h_{1}(\tau)-\lambda h_{2}(\tau)\right] R^{-1}(\tau) d \tau}
$$

Substituting Eq. (22) into Eq. (20) results in

$$
a_{M}(\tau)=\frac{f_{1}\left[h_{1}(\tau)-\lambda h_{2}(\tau)\right] R^{-1}(\tau)}{\int_{t}^{t_{f}} h_{1}(\tau)\left[h_{1}(\tau)-\lambda h_{2}(\tau)\right] R^{-1}(\tau) d \tau}
$$

which is an incomplete guidance command due to the unknown Lagrange multiplier $\lambda$.

From Eq. (19), it can be obtained that the minimum value of the performance index is given by

$$
\begin{aligned}
J & =\frac{\left(f_{1}-\lambda f_{2}\right)^{2}}{2 \int_{t}^{t_{f}}\left[h_{1}(\tau)-\lambda h_{2}(\tau)\right]^{2} R^{-1}(\tau) d \tau} \\
& =\frac{\left(f_{1}-\lambda f_{2}\right)^{2}}{2\left(g_{1}-2 \lambda g_{12}+\lambda^{2} g_{2}\right)}
\end{aligned}
$$

where

$$
\begin{aligned}
g_{1} & =\int_{t}^{t_{f}} h_{1}^{2}(\tau) R^{-1}(\tau) d \tau \\
g_{12} & =\int_{t}^{t_{f}} h_{1}(\tau) h_{2}(\tau) R^{-1}(\tau) d \tau \\
g_{2} & =\int_{t}^{t_{f}} h_{2}^{2}(\tau) R^{-1}(\tau) d \tau
\end{aligned}
$$

In order to find the optimal Lagrange multiplier $\lambda$ that gives the minimum value of the cost function, we impose the necessary condition $d J / d \lambda=0$ on Eq. (24) and then one can easily obtain

$$
\lambda=\frac{f_{1} g_{12}-f_{2} g_{1}}{f_{1} g_{2}-f_{2} g_{12}}
$$

Substituting Eq. (26) into Eq. (23) gives the generic optimal guidance command in a feedback form as

$$
a_{M}=N_{1} \frac{z}{t_{g o}^{2}}+N_{2} \frac{V_{M}\left(\gamma_{M}-\gamma_{f}^{d}\right)}{t_{g o}}
$$

where the navigation gains are determined by

$$
N_{1}=\frac{h_{1} g_{2}-h_{2} g_{12}}{g_{1} g_{2}-g_{12}^{2}} R^{-1} t_{g o}^{2}, \quad N_{2}=\frac{h_{2} g_{1}-h_{1} g_{12}}{\left(g_{1} g_{2}-g_{12}^{2}\right) V_{M}} R^{-1} t_{g o}
$$

To shape the guidance command, we consider a time-togo weighting function as $R^{-1}(t)=t_{g o}^{n}$ with $n \geq 0$ [15], [19]. Accordingly, if the value of $n$ is chosen as $n=0$, the resulting optimal guidance law is for energy consumption minimization. Additionally, we can safely predict that the designed guidance law with $n>0$ guarantees zero terminal acceleration command since the weighting function with $n>0$ becomes infinite as $t_{g o}$ approaches 0 . This property provides the missile with guaranteed operational margins to cope with the undesired disturbances when the missile approaches the target. It is also desirable to maximize the effectiveness of direct hit by reducing the shear angle. Obviously, one can utilize other weighting functions to further shape or distribute the guidance command during the terminal homing phase. Since the interceptor's velocity is time-varying, obtaining analytical solution of integration (25) is intractable. To address this problem, we will assume a constant-speed vehicle and update the velocity at every time instant when implementing the guidance law. For simplicity, the concept of average speed during the engagement is utilized here for guidance command derivation. Also, the average speed is assumed to be constant and updated at every time instant when computing the guidance gains. By using small angle approximation of shear 
angle, the average speed can be approximated by the definition as

$$
\bar{V}_{M} \triangleq \frac{1}{2}\left(V_{M}+V_{M_{f}}\right)=V_{M}+\frac{1}{2} a_{x} t_{g o}
$$

where the fact $V_{M_{f}}=V_{M}+a_{x} t_{g o}$ is used. By using the average speed, the term $h_{1}$ can be rewritten as

$$
h_{1}=\frac{\bar{V}_{M}}{V_{M}} t_{g o}
$$

substituting Eqs. (15) and (30) with $R^{-1}(t)=t_{g o}^{n}$ into Eq. (25) gives

$$
g_{1}=\frac{\bar{V}_{M}^{2}}{V_{M}^{2}} \frac{t_{g o}^{n+3}}{n+3}, \quad g_{12}=-\frac{\bar{V}_{M}}{V_{M}^{2}} \frac{t_{g o}^{n+2}}{n+2}, \quad g_{2}=\frac{1}{V_{M}^{2}} \frac{t_{g o}^{n+1}}{n+1}
$$

Substituting Eq. (31) into Eq. (28) yields the navigation gains as

$$
N_{1}=(n+2)(n+3) \frac{V_{M}}{\bar{V}_{M}}, \quad N_{2}=(n+1)(n+2)
$$

The explicit guidance command is then obtained by substituting Eq. (32) into Eq. (27). It is clear that the guidance command is given by a feedback form with a time-varying gain $N_{1}$ and a fixed gain $N_{2}$. Here, we can readily observe an interesting feature of time-varying $N_{1}$ : it is scaled by the ratio of the current speed over the average speed.

\section{Analysis of the Proposed Guidance LaW}

In this section, the capturability of the proposed optimal guidance law is analyzed first by utilizing the instantaneous linear time-invariant system concept. Then, the connection between the proposed guidance law with previous impact angle guidance laws is discussed.

\section{A. Capturability of the Proposed Guidance Law}

In order to provide better insights of the proposed guidance law, this subsection analyzes the capturability of the proposed guidance law. According to Eq. (10), the heading error can be

$$
e_{\gamma}=-\frac{z}{V_{M} t_{g o}+\frac{1}{2} a_{x} t_{g o}^{2}}=-\frac{z}{\bar{V}_{M} t_{g o}}
$$

Let $e_{I}=\gamma_{M}-\gamma_{f}^{d}$ be the intercept angle error. Differentiating Eq. (33) with respect to time and substituting Eq. (27) into it gives the heading error dynamics as

$$
\dot{e}_{\gamma}=-\frac{1}{t_{g o}}\left(N_{1} \frac{\bar{V}_{M}}{V_{M}}-1\right) e_{\gamma}+\frac{N_{2}}{t_{g o}} e_{I}
$$

The intercept angle error dynamics is obtained by substituting Eq. (27) into (13) as

$$
\dot{e}_{I}=-N_{1} \frac{\bar{V}_{M}}{V_{M} t_{g o}} e_{\gamma}+N_{2} \frac{e_{I}}{t_{g o}}
$$

The entire guidance loop dynamics is then derived by rearranging Eqs. (34) and (35) with Eq. (32) as

$$
\dot{x}=g(t) A x
$$

where

$$
x \triangleq\left[e_{\gamma}, e_{I}\right]^{T}, \quad g(t) \triangleq 1 / t_{g o}
$$

$$
A \triangleq\left[\begin{array}{cc}
-((n+2)(n+3)-1) & (n+1)(n+2) \\
-(n+2)(n+3) & (n+1)(n+2)
\end{array}\right]
$$

It is clear that system (36) is a special form of linear timevarying system, where $g(t)$ is a continuous function for all $t \geq 0$ and $A$ is a nonsigular constant matrix. According to the reference [19], the linear time-varying system shown in Eq. (36) is globally exponentially stable if $A$ is Hurwitz, that is all eigenvalues of $A$ are in the open left-half plane, and $g(t)$ is positive for all $t \geq 0$. First, we can easily know that $g(t)$ shown in Eq. (37) is always positive because $t_{g o}>0$ for all $t \geq 0$ by the definition. Next, the eigenvalues of $A$ are given by

$$
\lambda_{1}=-(n+2), \quad \lambda_{2}=-(n+1)
$$

From the preceding equation, we can readily observe that the eigenvalues of $A$ with $n \geq 0$ are always negative. Hence, the system (36) is globally exponentially stable. Since the equilibrium of system (36) is the origin, both the heading error $e_{\gamma}$ and the intercept angle error $e_{I}$ will exponentially converge to zero. By the definition of the instantaneous ZEM, it also converges to zero during the endgame, thus guaranteeing successful target capture with desired angles.

\section{B. Discussion of the Proposed Guidance Law}

From Eq. (32) with Eq. (29), it is clear that the initial value of time-varying navigation gain $N_{1}$ given by

$$
N_{1}(0)=(n+2)(n+3) \frac{V_{M 0}}{\left(V_{M 0}+\frac{1}{2} a_{x} t_{f}\right)}
$$

where $t_{f}$ represents the time of impact, $V_{M 0}$ the initial velocity of the interceptor. The final value of $N_{1}$ is determined by

$$
N_{1}\left(t_{f}\right)=(n+2)(n+3)
$$

Through simple algebra, it is easy to verify that

$$
N_{1}(0)<N_{1}(t)<N_{1}\left(t_{f}\right), \quad \forall t \in\left(0, t_{f}\right)
$$

which reveals that the navigation gain $N_{1}$ monotonically increases and converges to constant value at the time of impact. This provides the unique information that how the navigation gain $N_{1}$ behaves as the vehicle's speed changes during the terminal guidance phase. That is, only when the interceptor is close to the target, the effect of the speed variation can be ignored. Therefore, it is safe to predict that classical optimal impact angle guidance laws with constant navigation gains are not 'real optimal' in a realistic exo-atmospheric engagement.

In [25], we reveal that, if we remove the gravitational effect, the instantaneous ZEM reduces to the ZEM, denoted as $z_{G 2 C}$, that is used for guidance-to-collision (G2C) guidance law [10], [21] derivation. The dynamics of $z_{G 2 C}$ is given by [25]

$$
\dot{z}_{G 2 C}=-a_{M}\left(t_{g o}+\frac{a_{x}}{2 V_{M}} t_{\text {go }}^{2}\right)
$$

Under this condition, it is easy to verify that the proposed guidance law becomes

$$
a_{M}=N_{1} \frac{z_{G 2 C}}{t_{g o}^{2}}+N_{2} \frac{V_{M}\left(\gamma_{M}-\gamma_{f}^{d}\right)}{t_{g o}}
$$


which has the same navigation gains as the proposed guidance law but with different ZEM definition. Since the time-to-go weighted cost function is utilized to derive the guidance law, we call this guidance law as time-to-go weighted optimal G2C impact angle guidance (TWOG2CIAG). Note that this is also a new guidance law that has never been proposed in existing works.

In practical implementation, the TWOG2CIAG requires an extra term to reject the effect of gravity as

$$
a_{M}=N_{1} \frac{z_{G 2 C}}{t_{g o}^{2}}+N_{2} \frac{V_{M}\left(\gamma_{M}-\gamma_{f}^{d}\right)}{t_{g o}}+g \cos \gamma_{M}
$$

which might require more control energy and lose some control operational margins and direct hit effectiveness.

If we assume constant-speed vehicle under gravity-free condition, the instantaneous ZEM will converge to the classical ZEM, denoted as $z_{P N G}$, utilized for proportional navigation guidance (PNG) derivation. The dynamics of $z_{P N G}$ is determined by [15]

$$
\dot{z}_{P N G}=-a_{M} t_{g o}
$$

Using $z_{P N G}$, the proposed optimal guidance law reduces to

$$
\begin{aligned}
a_{M}= & (n+2)(n+3) \frac{z_{P N G}}{t_{g o}^{2}} \\
& +(n+1)(n+2) \frac{V_{M}\left(\gamma_{M}-\gamma_{f}^{d}\right)}{t_{g o}}
\end{aligned}
$$

which is an exact equivalent form of the TWOIAG proposed in [18], [19] (proof of the equivalence is given in Appendix A). This clearly shows that the navigation gains of the proposed guidance converge to those of the TWOIAG at the time of impact and hence the proposed guidance law exhibits similar characteristics as TWOIAG when the missile approaches the target or PIP.

Similarly, since the TWOIAG is derived without the consideration of gravity, it requires an extra term to compensate for the gravity as

$$
\begin{aligned}
a_{M}= & (n+2)(n+3) \frac{z_{P N G}}{t_{g o}^{2}} \\
& +(n+1)(n+2) \frac{V_{M}\left(\gamma_{M}-\gamma_{f}^{d}\right)}{t_{g o}}+g \cos \gamma_{M}
\end{aligned}
$$

which cannot guarantee zero final guidance command, leading to the sacrifice of operational gains against unexpected disturbances as well as the direct hit effectiveness. As a comparison, the proposed guidance law utilizes the instantaneous ZEM, which automatically includes the effect of the gravity. This means that no extra gravity-compensation term is required for practical implementation of the proposed approach and zero final guidance command is theoretically guaranteed.

Additionally, since TWOIAG is derived under constant velocity assumption, it cannot drive the missile to follow the desired straight line collision course and is far away from optimal for exo-atmospheric interception scenarios. Compared with the constant navigation gains that used in the TWOIAG, the proposed approach leverages time-varying navigation gain
TABLE I

INITIAL CONDITIONS FOR HOMING ENGAGEMENT.

\begin{tabular}{cc}
\hline \hline Parameters & Values \\
\hline Missile-target initial relative range, $r(0)$ & $50 \mathrm{~km}$ \\
\hline Initial LOS angle, $\lambda(0)$ & $0^{\circ}$ \\
\hline Missile initial velocity, $V_{M}(0)$ & $2500 \mathrm{~m} / \mathrm{s}$ \\
\hline Missile initial flight path angle, $\gamma_{M}(0)$ & $150^{\circ}$ \\
\hline Missile axial acceleration, $a_{x}$ & $20 \mathrm{~g}$ \\
\hline Target velocity, $V_{T}$ & $3000 \mathrm{~m} / \mathrm{s}$ \\
\hline Target initial flight path angle, $\gamma_{T}(0)$ & $20^{\circ}$ \\
\hline Desired Intercept Angle, $\gamma_{f}^{d}$ & $160^{\circ}$ \\
\hline \hline
\end{tabular}

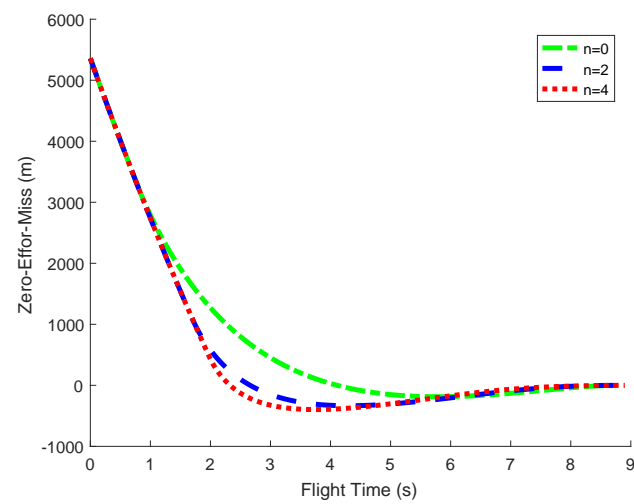

Fig. 3. Instantaneous zero-effort-miss profile.

that is dependent on the missile's velocity and axial acceleration. This fact shows that how the proposed guidance law can counteract the effect of speed variation, as discussed before. In conclusion, the proposed optimal guidance law is a general formulation, that encompasses both TWOIAG and TWOG2CIAG, and is more suitable for practical applications.

\section{Simulation Results}

In this section, nonlinear numerical simulations are performed to validate the proposed optimal guidance law in an exo-atmospheric interception scenario. The required initial conditions are summarized in Table I. We first analyze the basic properties of the proposed guidance law with different $n$ and then make comparisons with TWOIAG and TWOG2CIAG.

\section{A. Characteristics of the Proposed Guidance Law}

This subsection analyzes the characteristics of the proposed guidance law with various design parameters $n=0,2,4$. Figures 3 and 4 provide the simulation results of instantaneous ZEM and flight path angle obtained by the proposed guidance law. It is clear that both the instantaneous ZEM and intercept angle error converge to zero at the time of impact, meaning that the proposed guidance law can successfully guide the missile to intercept the target with desired terminal angles once $n \geq 0$. One can also note from these two figures that increasing the cost function order $n$ generates faster convergence of the system states when the missile approaches the target. The shear angle commands with different $n$ are depicted in Fig. 5. This figure reveals that the proposed guidance law with 


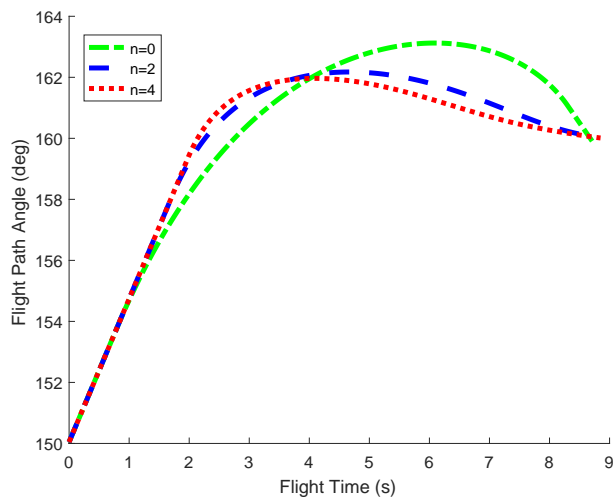

Fig. 4. Flight path angle history.

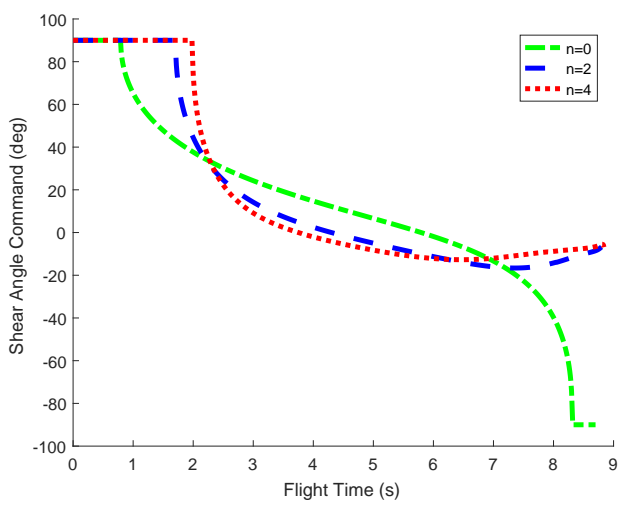

Fig. 5. Shear angle command.

$n \geq 1$ ensures zero guidance command at the time of impact, as we expected. Increasing $n$ requires more control energy during the initial phase to drive the missile to the desired collision triangle. Figure 6 compares the missile velocity under the proposed guidance law with various $n$. The results reveal that the proposed guidance law initially utilizes all available energy to drive the interceptor to converge to ideal collision triangle and maintains close to the collision triangle thereafter. Therefore, the missile velocity under the proposed guidance law increases almost linearly once the collision triangle is achieved. However, guidance law with $n=0$ cannot guarantee zero final guidance command and the shear angle appears to be saturated when the missile is near the target. Therefore, the vehicle's speed with $n=0$ remains constant during that period.

\section{B. Comparison with Other Guidance Laws}

To show the superiority of the proposed guidance law, both TWOIAG and TWOG2CIAG are also performed in the simulation for the purpose of comparison. These two guidance laws are implemented by adding a gravity compensation term. To make fair comparisons, the time-to-go order of all guidance laws are chosen as $n=3$.

Figure 7 shows the simulation results of interception trajectories of different guidance laws. This figure reveals that these three guidance laws can drive the missile to intercept the

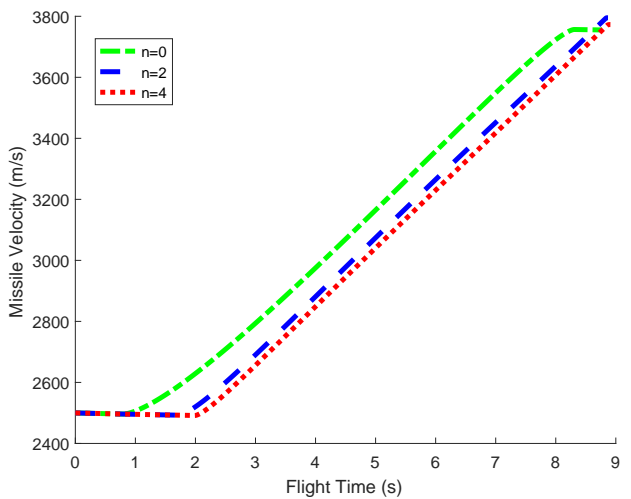

Fig. 6. Missile velocity profile.

target at some extent, but the trajectories under TWOIAG is more curved. The time history of the flight angle is presented in Fig. 8, which clearly shows that the proposed guidance law and TWOG2CIAG have higher accuracy in driving the intercept angle error to zero than that of TWOIAG. This validates the benefits of utilizing time-varying navigation gains in the TWOG2CIAG and proposed framework, compared to the constant gains in TWOIAG. Figure 9 presents the ZEM time histories under different guidance laws, showing that all guidance laws can ensure zero final ZEM to guarantee successful interception. The comparison of shear angle guidance commands of different guidance laws is given in Fig. 10. As we stated earlier, by choosing $n>0$, the proposed optimal guidance law guarantees zero guidance command at the time of impact since the instantaneous ZEM automatically contains the effect of the gravitational effect. This can be clearly observed from Fig. 10. As a comparison, the shear angle command under TWOIAG saturates during the last 2 seconds. Therefore, the proposed guidance law can improve the effectiveness of direct hit compared with the other guidance laws. Additionally, the proposed guidance has more operational margins to cope with the undesired disturbances when the missile approaches the target. This fact is of great importance in practical applications to reduce the terminal miss distance and intercept angle error. Compared to the proposed approach, the TWOG2CIAG requires more energy during the initial phase and only guarantee bounded terminal guidance command. The velocity profile, presented in Fig. 11, demonstrates that the proposed guidance law has the highest terminal impact velocity compared to the other two laws, leading to the increase of final impact energy. Since the guidance command of TWOIAG saturates when the missile approaches the target, the missile velocity remains constant during that period.

The quantitative comparison results regarding miss distance, intercept angle error and control effort are summarized in Table II, where the control effort is defined as $\int_{t_{0}}^{t} a_{M}^{2}(\tau) d \tau$. By comparing TWOG2CIAG with TWOIAG, the results shown in Table II reveal that utilizing time-varying navigation gain helps to reduce the control energy and increase the guidance accuracy. It is also clear that using gravity-turn, instead of compensating for it, in the proposed guidance law can save 


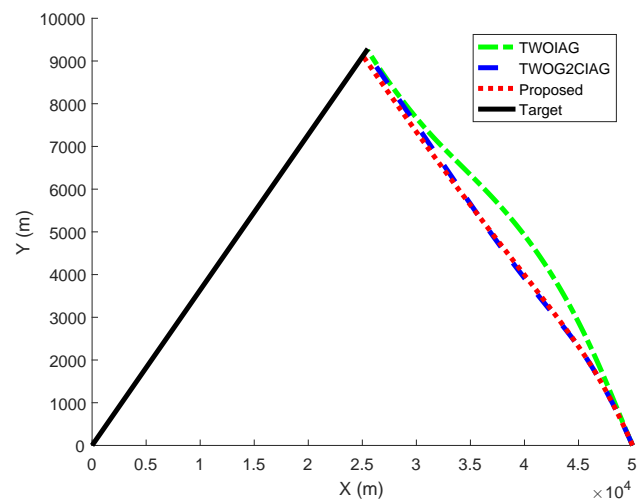

Fig. 7. Interception trajectory.

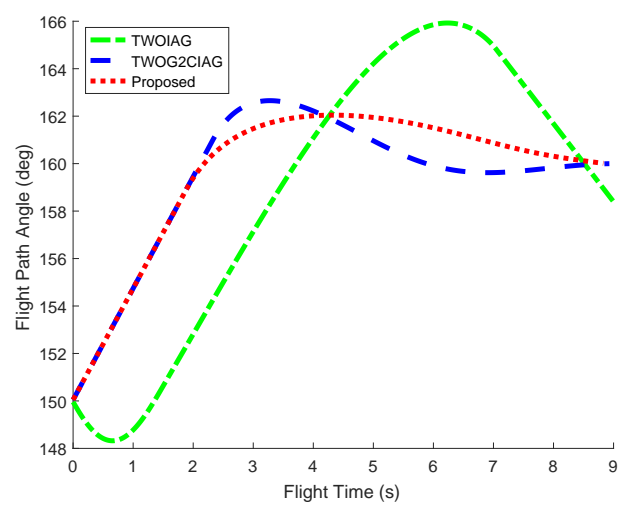

Fig. 8. Flight path angle profile.

energy consumption and slightly increase the interception accuracy, as compared with TWOG2CIAG. In conclusion, the proposed guidance law exhibits better overall performance than the other two guidance laws in terms of control effort and guidance accuracy.

\section{Conclusions}

This paper proposes a novel optimal intercept angle guidance for exo-atmospheric interception. The proposed guidance law is given in a feedback form in terms of the instantaneous

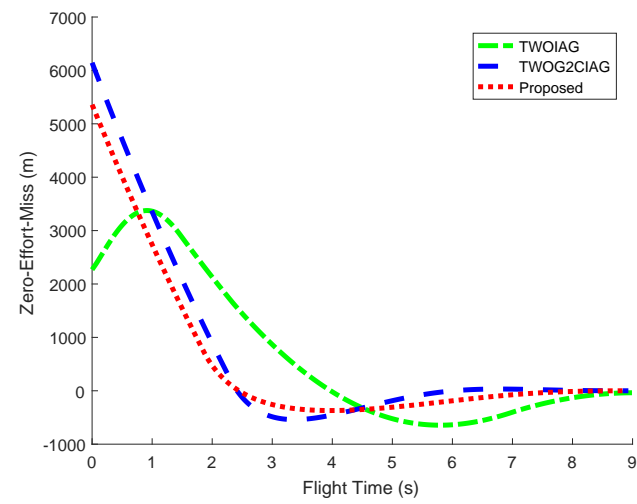

Fig. 9. Zero-effort-miss.

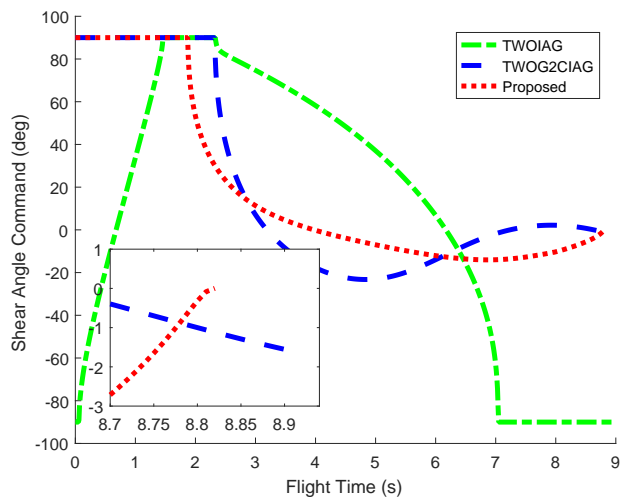

Fig. 10. Shear angle guidance command.

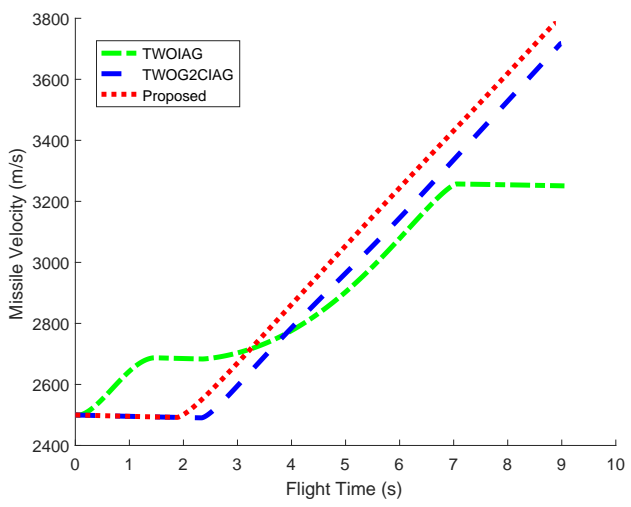

Fig. 11. Missile velocity profile.

ZEM and intercept angle error with time-varying navigation gain. The uniqueness of the proposed approach is that it directly uses, instead of compensating for, the gravity and also accommodate the speed-varying issue. Theoretical analysis shows that both TWOIAG and TWOG2CIAG are special cases of the proposed guidance law. Compared with TWOIAG and TWOG2CIAG, the proposed guidance law has better performance in terms of energy consumption and guidance accuracy. It should be noted that the proposed approach can also be utilized in mid-course guidance as the angle constraint is important for guaranteeing the lock-on condition of the onboard seekers.

TABLE II

QUANTITATIVE COMPARISON RESULTS.

\begin{tabular}{c|ccc}
\hline \hline & TWOIAG & TWOG2CIAG & Proposed \\
\hline Miss distance & $5.771 \mathrm{~m}$ & $0.940 \mathrm{~m}$ & $0.931 \mathrm{~m}$ \\
\hline Intercept angle error & $1.66^{\circ}$ & $0.88^{\circ}$ & $0.88^{\circ}$ \\
\hline Control effort & 228575 & 109008 & 91503 \\
\hline \hline
\end{tabular}




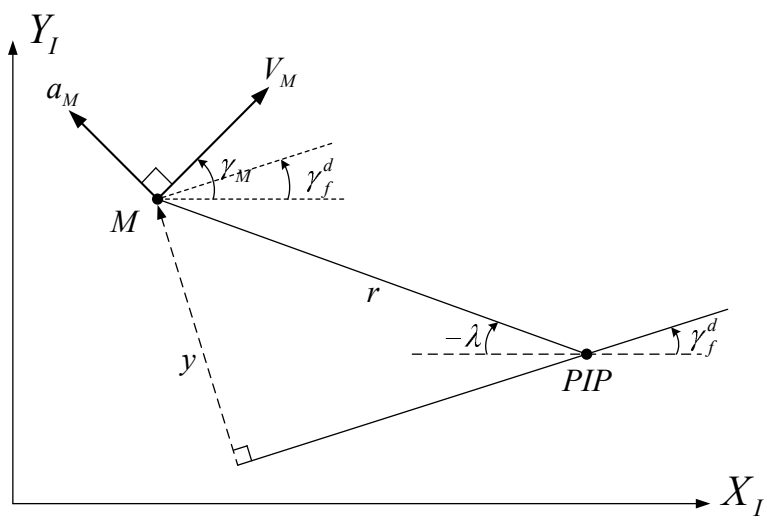

Fig. 12. The homing engagement geometry .

\section{APPENDIX A \\ APPENDIX: EQUiVAlent Form OF TIME-TO-Go \\ Weighted Optimal IMPACT ANGLE Guidance}

The guidance command of TWOIAG is given by [18], [19]

$$
a_{M}=-\frac{(n+2)(n+3)}{t_{g o}^{2}} y-\frac{2(n+2)}{t_{g o}} v, \quad n \geq 0
$$

where $y$ is vertical relative distance to the impact frame with respect to the PIP, as shown in Fig. 12, and $v=\dot{y}$.

Under the assumption that the angle $\bar{\gamma}_{M}=\gamma_{M}-\gamma_{f}^{d}$ is small, the kinematics can be obtained as

$$
\dot{y}=v=V_{M} \bar{\gamma}_{M}
$$

The flight path angle and LOS angle in the linearized engagement kinematics as shown in Fig. 12 can be expressed in terms of $y$ and $v$ from the engagement geometry as

$$
\bar{\gamma}_{M}=\frac{v}{V_{M}}, \quad \bar{\lambda}=\lambda-\gamma_{f}^{d}=-\frac{y}{r}
$$

The relative range between the PIP and the missile is approximated by multiplying the missile velocity by time-togo as $r \approx V_{M} t_{g o}$. Then, one can imply that

$$
y=V_{M} t_{g o}\left(\gamma_{f}^{d}-\lambda\right), \quad v=V_{M}\left(\gamma_{M}-\gamma_{f}^{d}\right)
$$

which represents the relationship between the parameters of the linearized engagement kinematics (i.e., $y$ and $v$ ) and the parameters of the nonlinear engagement kinematics (i.e., $\lambda$ and $\left.\gamma_{M}\right)$.

Rearranging guidance command (49) and substituting (52) into it yields

$$
\begin{aligned}
a_{M}= & -\frac{(n+2)(n+3)}{t_{g o}^{2}}\left(y+v t_{g o}\right) \\
& +\frac{(n+1)(n+2)}{t_{g o}} V_{M}\left(\gamma_{M}-\gamma_{f}^{d}\right)
\end{aligned}
$$

In the linearized engagement considered in Fig. 12, the ZEM that used in PNG derivation is given by [15]

$$
z_{P N G}=-\left(y+v t_{g o}\right)
$$

Substituting (54) into (53) gives the guidance command as

$$
a_{M}=(n+2)(n+3) \frac{z_{P N G}}{t_{g o}^{2}}+(n+1)(n+2) \frac{V_{M}\left(\gamma_{M}-\gamma_{f}^{d}\right)}{t_{g o}}
$$

\section{REFERENCES}

[1] B.-G. Park, T.-H. Kim, and M.-J. Tahk, "Range-to-go weighted optimal guidance with impact angle constraint and seeker's look angle limits," IEEE Transactions on Aerospace and Electronic Systems, vol. 52, no. 3, pp. 1241-1256, 2016.

[2] Z. Hou, L. Liu, Y. Wang, J. Huang, and H. Fan, "Terminal impact angle constraint guidance with dual sliding surfaces and model-free target acceleration estimator," IEEE Transactions on Control Systems Technology, vol. 25, no. 1, pp. 85-100, 2017.

[3] A. Ratnoo and D. Ghose, "State-dependent riccati-equation-based guidance law for impact-angle-constrained trajectories," Journal of Guidance, Control, and Dynamics, vol. 32, no. 1, pp. 320-325, 2009.

[4] S. R. Kumar, S. Rao, and D. Ghose, "Sliding-mode guidance and control for all-aspect interceptors with terminal angle constraints," Journal of Guidance, Control and Dynamics, vol. 35, no. 4, pp. 1230-1246, 2012.

[5] — "Nonsingular terminal sliding mode guidance with impact angle constraints," Journal of Guidance, Control, and Dynamics, vol. 37, no. 4, pp. 1114-1130, 2014.

[6] S. Rao and D. Ghose, "Terminal impact angle constrained guidance laws using variable structure systems theory," IEEE Transactions on Control Systems Technology, vol. 21, no. 6, pp. 2350-2359, 2013.

[7] C.-H. Lee, T.-H. Kim, and M.-J. Tahk, "Design of impact angle control guidance laws via high-performance sliding mode control," Proceedings of the Institution of Mechanical Engineers, Part G: Journal of Aerospace Engineering, vol. 227, no. 2, pp. 235-253, 2013.

[8] S. He, D. Lin, and J. Wang, "Continuous second-order sliding mode based impact angle guidance law," Aerospace Science and Technology, vol. 41, pp. 199-208, 2015.

[9] S. He, T. Song, and D. Lin, "Impact angle constrained integrated guidance and control for maneuvering target interception," Journal of Guidance, Control, and Dynamics, vol. 40, no. 10, pp. 2653-2661, 2017.

[10] D. Reisner and T. Shima, "Optimal guidance-to-collision law for an accelerating exoatmospheric interceptor missile," Journal of Guidance, Control, and Dynamics, vol. 36, no. 6, pp. 1695-1708, 2013.

[11] T. Shima, "Intercept-angle guidance," Journal of Guidance Control and Dynamics, vol. 34, no. 2, pp. 484-492, 2011.

[12] S. He and C.-H. Lee, "Optimality of error dynamics in missile guidance problems," Journal of Guidance, Control, and Dynamics, vol. 41, no. 7, pp. 1624-1633, 2018.

[13] M. Kim and K. V. Grider, "Terminal guidance for impact attitude angle constrained flight trajectories," IEEE Transactions on Aerospace and Electronic Systems, no. 6, pp. 852-859, 1973.

[14] T. L. Song and S. J. Shin, "Time-optimal impact angle control for vertical plane engagements," IEEE Transactions on Aerospace and Electronic Systems, vol. 35, no. 2, pp. 738-742, 1999.

[15] P. Zarchan, Tactical and strategic missile guidance. American Institute of Aeronautics and Astronautics, 2012.

[16] C.-K. Ryoo, H. Cho, and M.-J. Tahk, "Optimal guidance laws with terminal impact angle constraint," Journal of Guidance Control and Dynamics, vol. 28, no. 4, pp. 724-732, 2005.

[17] V. Shaferman and T. Shima, "Linear quadratic guidance laws for imposing a terminal intercept angle," Journal of Guidance, Control, and Dynamics, vol. 31, no. 5, pp. 1400-1412, 2008.

[18] E. J. Ohlmeyer and C. A. Phillips, "Generalized vector explicit guidance," Journal of Guidance, Control, and Dynamics, vol. 29, no. 2, pp. 261-268, 2006.

[19] C.-K. Ryoo, H. Cho, and M.-J. Tahk, "Time-to-go weighted optimal guidance with impact angle constraints," IEEE Transactions on Control Systems Technology, vol. 14, no. 3, pp. 483-492, 2006.

[20] C.-H. Lee, M.-J. Tahk, and J.-I. Lee, "Generalized formulation of weighted optimal guidance laws with impact angle constraint," IEEE Transactions on Aerospace and Electronic Systems, vol. 49, no. 2, pp. 1317-1322, 2013.

[21] T. Shima and O. M. Golan, "Exo-atmospheric guidance of an accelerating interceptor missile," Journal of the Franklin Institute, vol. 349, no. 2, pp. 622-637, 2012.

[22] R. Gazit and S. Gutman, "Development of guidance laws for a variablespeed missile," Dynamics and Control, vol. 1, no. 2, pp. 177-198, 1991.

[23] H. Cho and C. K. Ryoo, "Implementation of optimal guidance laws using predicted missile velocity profiles," Journal of Guidance, Control, and Dynamics, vol. 22, no. 4, pp. 579-588, 1999.

[24] H. Cho, C. K. Ryoo, and M.-J. Tahk, "Closed-form optimal guidance law for missiles of time-varying velocity," Journal of Guidance, Control, and Dynamics, vol. 19, no. 5, pp. 1017-1022, 1996. 
[25] S. He and C.-H. Lee, "Gravity-turn-assisted optimal guidance law," Journal of Guidance, Control, and Dynamics, vol. 41, no. 1, pp. 171183,2018

[26] M.-G. Seo, C.-H. Lee, and M.-J. Tahk, "New design methodology for impact angle control guidance for various missile and target motions," IEEE Transactions on Control Systems Technology, 2017.

[27] H. B. Oza and R. Padhi, "Impact-angle-constrained suboptimal model predictive static programming guidance of air-to-ground missiles," Journal of Guidance, Control, and Dynamics, vol. 35, no. 1, pp. 153-164, 2012.

[28] J.-H. Kim, I. H. Whang, and B. M. Kim, "Finite horizon integrated guidance and control for terminal homing in vertical plane," Journal of Guidance, Control, and Dynamics, vol. 39, no. 5, pp. 1104-1112, 2016.

[29] X. Bu, X. Wu, M. Tian, J. Huang, R. Zhang, and Z. Ma, "Highorder tracking differentiator based adaptive neural control of a flexible air-breathing hypersonic vehicle subject to actuators constraints," ISA transactions, vol. 58, pp. 237-247, 2015.

[30] X. Bu, X. Wu, D. Wei, and J. Huang, "Neural-approximation-based robust adaptive control of flexible air-breathing hypersonic vehicles with parametric uncertainties and control input constraints," Information Sciences, vol. 346, pp. 29-43, 2016.

[31] R. Padhi and M. Kothari, "Model predictive static programming: a computationally efficient technique for suboptimal control design," International Journal of Innovative Computing, Information and Control, vol. 5, no. 2, pp. 399-411, 2009.

[32] S. Han, M.-C. Hwang, B.-Y. Lee, J. Ahn, and M.-J. Tahk, "Analytic solution of projectile motion with quadratic drag and unity thrust," IFACPapersOnLine, vol. 49, no. 17, pp. 40-45, 2016.

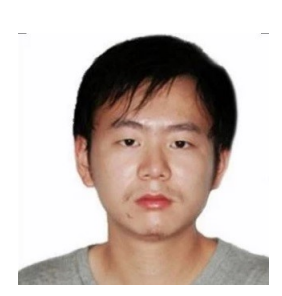

Shaoming He was born in 1991. He received his $\mathrm{MSc}$ degree in Aerospace Engineering from Beijing Institute of Technology in 2016. He is currently a PhD student in the School of Aerospace, Transport and Manufacturing, Cranfield University. His research interests include multi-target tracking, guidance and control.

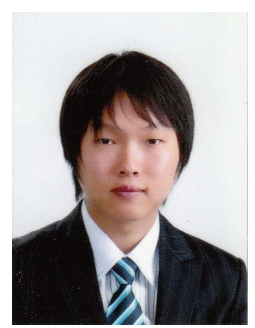

Chang-Hun Lee received the B.S., M.S., and $\mathrm{Ph} . \mathrm{D}$. degrees in aerospace engineering from Korea Advanced Institute of Science and Technology (KAIST), in 2008, 2010, and 2013, respectively.

From 2013 to 2015 he was a senior researcher for guidance and control (G\&C) team at the Agency for Defense Development (ADD) in Korea. Since 2016, he has worked for school of aerospace, transportation, and manufacturing at Cranfield University, UK where he is currently a research fellow. His recent research areas are advanced missile guidance and control, cooperative control for UAVs, target tracking filter, deep learning, and aviation data analytics. 
2018-09-24

\section{Optimal impact angle guidance for exo-atmospheric interception utilizing gravitational effect}

He, Shaoming

IEEE

Shaoming He and Chang-Hun Lee. Optimal impact angle guidance for exo-atmospheric interception utilizing gravitational effect. IEEE Transactions on Aerospace and Electronic Systems, Volume 55, Issue 3, 2019, pp. 1382-1392

https://doi.org/10.1109/TAES.2018.2870456

Downloaded from Cranfield Library Services E-Repository 